\title{
Adrenocortical Causes of Hypertension
}

\author{
Andreas Moraitis and Constantine Stratakis \\ Eunice Kennedy Shriver National Institute of Child Health and Human Development, \\ Program on Developmental Endocrinology and Genetics, Bethesda, MD 20892, USA \\ Correspondence should be addressed to Constantine Stratakis, stratakc@mail.nih.gov
}

Received 18 November 2010; Revised 9 January 2011; Accepted 13 January 2011

Academic Editor: Vasilios Papademetriou

Copyright (C) 2011 A. Moraitis and C. Stratakis. This is an open access article distributed under the Creative Commons Attribution License, which permits unrestricted use, distribution, and reproduction in any medium, provided the original work is properly cited.

\begin{abstract}
Primary aldosteronism is the most common cause of secondary hypertension. In the past, screening for primary aldosteronism was offered only in patients with hypertension associated with hypokalemia. Recent studies showed that hypokalemia is seen in only $25 \%$ of the patients with primary aldosteronism, which has increased the prevalence of primary aldosteronism to $10-15 \%$ of all cases with new onset hypertension.
\end{abstract}

\section{Introduction}

Approximately 85 million people in the United States are estimated to be hypertensive. In the majority, the hypertension is "essential" or "idiopathic," but a subgroup of approximately $15 \%$ has secondary hypertension. The secondary causes of hypertension can be divided into renal (e.g., renovascular or renal parenchymal disease) and endocrine causes. There are at least 14 endocrine disorders in which hypertension may be the initial clinical presentation (Table 1).

A further classification of the adrenocortical causes of hypertension based on the levels of renin and aldosterone can be used for the diagnostic approach of a patient with suspected endocrine hypertension (Table 2).

An accurate diagnosis of endocrine hypertension provides the clinician with a unique treatment opportunity, that is, to render a surgical cure or to achieve a dramatic response with pharmacologic therapy. Primary hyperaldosteronism, which represents the most common cause of endocrine resistant hypertension, is reviewed here.

\section{Aldosterone Biosynthesis and Control of Secretion}

The differentiation of the adrenal cortex into distinct zones has important functional consequences. The zona glomerulosa comprises approximately 15\% of the cortex (depending upon sodium intake). Cells are clustered in spherical nests and are small with smaller nuclei in comparison to other zones. The zona fasciculata comprises the $75 \%$ of the cortex; in this zone, the cells are large and lipid laden and form radial cords between the fibrovascular radial network. The innermost zona reticularis is sharply demarcated from both zona fasciculata and the adrenal medulla; cells are irregular with little lipid content. Three main types of hormone are produced by the adrenal cortex-glucocorticoids (cortisol, corticosterone), mineralocorticoids (aldosterone, deoxycorticosterone), and sex steroids (mainly androgens).

Aldosterone is synthesized from cholesterol in a series of six biosynthetic steps [1]. The first four steps are also involved in the synthesis of cortisol, whereas the last two pertain only to aldosterone [2]. The product of the CYP11B2 gene is capable of catalyzing both the 11-hydroxylase and 18-hydroxylase and 18-hydroxydehydrogenase steps in aldosterone biosynthesis [2-4]. The CYP11B2 gene is located on human chromosome 8q24.3-tel [5].

Aldosterone, the major circulating mineralocorticoid, is a steroid hormone produced exclusively in the zona glomerulosa. The major regulators of aldosterone biosynthesis and secretion are the renin-angiotensin system and potassium ion concentrations. Minor regulators include corticotrophin (ACTH) from the pituitary, atrial natriuretic peptide from the heart, and dopamine secreted locally in the adrenal. A number of aldosterone precursors, 
TABLE 1: Endocrine causes of hypertension.

\begin{tabular}{l}
\hline Adrenal dependent \\
\hline (1) Pheochromocytoma \\
(2) Cushing's syndrome \\
(3) Primary hyperaldosteronism \\
(4) Other adrenocortical tumors (i.e., carcinoma, other) \\
(5) Genetic defects affecting adrenocortical function \\
(i) Congenital adrenal hyperplasia: $11 \beta$-Hydroxylase and $17 \alpha$-Hydroxylase deficiency, primarily \\
$\quad$ ii) Primary cortisol resistance \\
Apparent mineralocorticoid excess (AME)/11 $\beta$-Hydroxysteroid dehydrogenase deficiency \\
Genetic \\
(i) Type 1 AME \\
(ii) Type 2 AME \\
Acquired \\
(i) Licorice or carbenoxolone ingestion (type 1 AME) \\
(ii) Cushing's syndrome (type 2 AME) \\
\hline Thyroid dependent \\
Hyperthyroidism \\
\hline Parathyroid dependent \\
Hyperparathyroidism \\
\hline Pituitary dependent \\
(1) Acromegaly \\
(2) Cushing's disease
\end{tabular}

TABLe 2: Adrenocortical causes of hypertension.

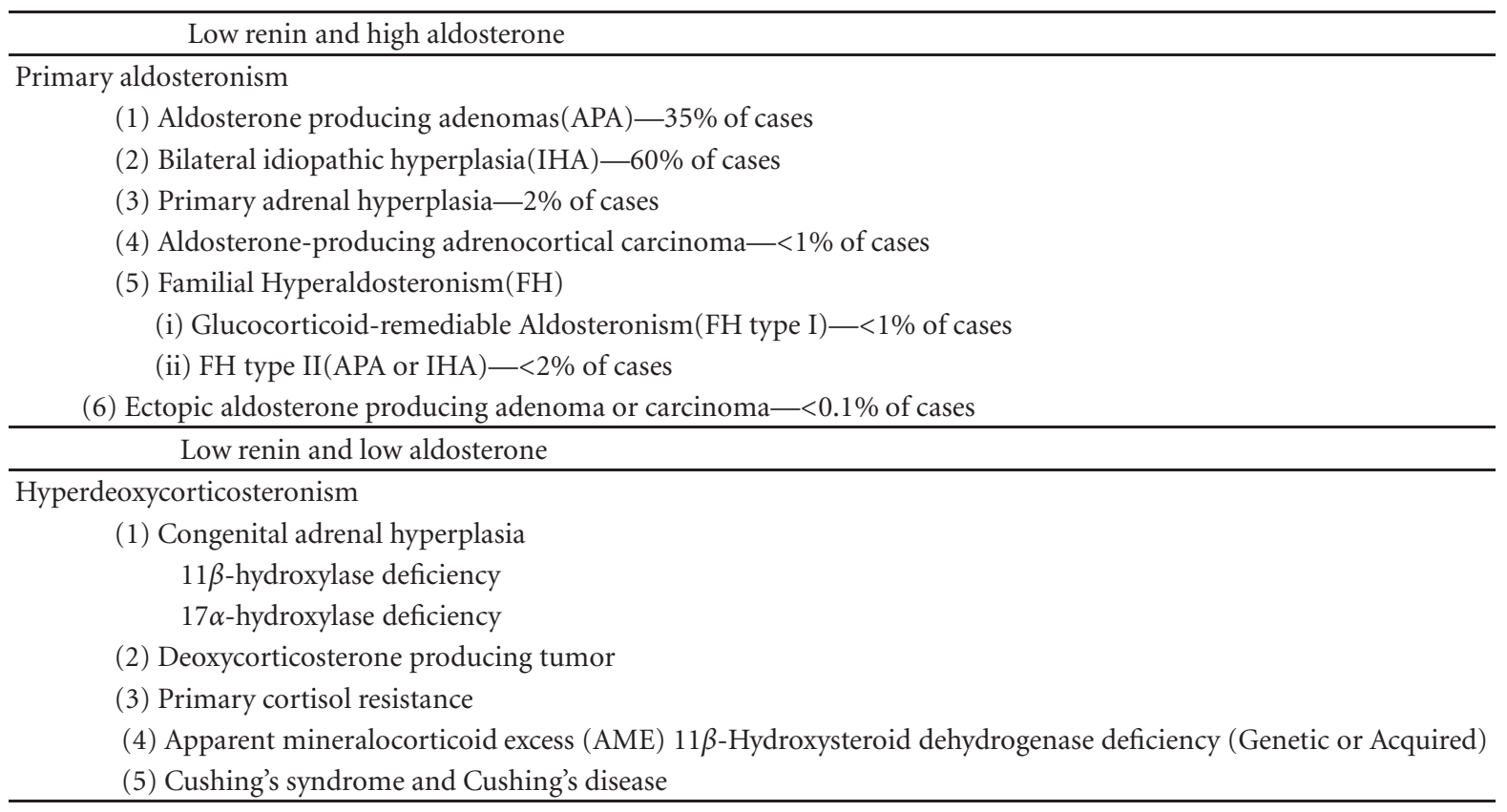

including deoxycorticosterone and 18-hydroxycorticosterone, have mineralocorticoid activity, and their hypersecretion in various pathological states may produce or exacerbate features typical of mineralocorticoid hypertension. Aldosterone acts mainly on the distal nephron although several other sites of sodium reabsorption exist.
The classic functions of aldosterone are regulation of extracellular volume and control of potassium homeostasis. These effects are mediated by binding of free aldosterone to the mineralocorticoid receptor in the cytosol of epithelial cells principally in the kidney. Mineralocorticoid receptors have a tissue-specific expression. For example, tissues with 
the highest concentration of these receptors are the distal nephron, hippocampus, and colon. Lower concentrations are found in the rest of the gastrointestinal tract and heart. Transport to the nucleus and binding to specific binding domains on targeted genes lead to their increased expression. Aldosterone-regulated kinase appears to be a key intermediary, and its increased expression leads to modification of the apical sodium channel, resulting in increased sodium ion transport across the cell membrane. The increased tubular negativity augments tubular secretion of potassium by the tubular cells and hydrogen ion by the interstitial cells.

Glucocorticoids and mineralocorticoids bind equally to the mineralocorticoid receptor. Specificity of action is provided by the presence of a glucocorticoid metabolizing enzyme, 11 $\beta$-hydroxysteroid dehydrogenase (11BHSD2), which metabolizes cortisol to cortisone and prevents glucocorticoids from interacting with the receptor.

In addition to the classic genomic actions mediated by aldosterone binding to cytosolic receptors, mineralocorticoids have acute, nongenomic actions due to activation of an unidentified cell surface receptor. This action involves most likely a $G$ protein-coupled receptor and probably a modification of the sodium hydrogen exchange activity.

Aldosterone has additional, nonclassic effects primarily on non epithelial cells. These include the expression of several collagen genes, genes controlling tissue growth factors, such as transforming growth factor $\beta$ and plasminogen activator inhibitor type 1 , and genes mediating inflammation. The resultant actions lead to microangiopathy, necrosis (acutely), and fibrosis in various tissues such as the heart, the vasculature, and the kidney.

At the level of the zona glomerulosa, the major stimulatory influences are angiotensin II and serum potassium $[6,7]$. ACTH stimulates aldosterone secretion in an acute but transient fashion, but it is questionable whether ACTH plays a significant role in the chronic regulation of mineralocorticoid secretion [8]. The major inhibitory influences affecting the zona glomerulosa are exerted by circulating atrial natriuretic peptide (ANP) and locally by dopamine [9]. Although ANP levels are clearly increased in hyperaldosteronism, neither ANP nor dopamine has been implicated as primary causes of clinically significant defects in aldosterone secretion. Metoclopramide increases aldosterone secretion, suggesting dopamine may inhibit aldosterone release [1012]. The physiologic roles of adrenomedullin and vasoactive intestinal peptide (VIP) on aldosterone secretion remain to be clarified; it appears that both these neuropeptides are produced in the rat zona glomerulosa $[13,14]$.

\section{Primary Hyperaldosteronism}

In his presidential address at the Annual Meeting of the Central Society for Clinical research, Chicago Illinois, October 29 1954, Dr. Jerome W. Conn stated: "I have prepared no comprehensive review of my personal philosophy of clinical investigation. Instead, I plan to make a scientific report to you about a clinical syndrome, the investigation of which has been most exciting to me since I initiated it in April of this year".

In April 1954, Conn was asked to see M. W., a 34-year-old woman with a 7-year history of muscle spasms, temporary paralysis, tetany, and weakness and a 4-year history of hypertension. Because there were no signs or symptoms of glucocorticoid or androgen excess, Conn suspected based on his prior research that M. W.'s clinical presentation could result from excess secretion of the adrenal salt-retaining corticoid. Conn planned for a bilateral adrenalectomy on December 1954. In 1955, Gittler and Fajans told of the surgical scene: To "the immense delight of Conn and those in the operating room, the surgeon, Dr. William Baum, encountered a right $13 \mathrm{gr}$ adrenal tumor which was removed while leaving the contralateral gland intact. The patient's post operative studies showed an almost total reversal of the preoperative metabolic and clinical abnormalities".

By 1964, Conn had collected 145 cases, and he suggested that up to $20 \%$ of patients with essential hypertension might have primary aldosteronism. Later, Conn decreased his predicted prevalence or primary aldosteronism to $10 \%$ of hypertensives, a prediction that was substantiated nearly 40 years later.

In the past, clinicians would not consider the diagnosis of primary hyperaldosteronism unless the patient presented with spontaneous hypokalemia, and then the diagnostic evaluation would require discontinuation of antihypertensive medications for at least 2 weeks. The spontaneous hypokalemia/no antihypertensive drug approach resulted in predicted prevalence rates of less than $0.5 \%$ of hypertensive patients.

However, it is now recognized that most patients with primary aldosteronism are not hypokalemic and that screening can be completed with a simple blood test (plasma aldosterone concentration[PAC]-to-plasma renin activity (PRA) ratio) while the patient is taking antihypertensive drugs. Using the PAC/PRA ratio as a screening test, followed by confirmatory testing, has resulted in much higher prevalence estimates (5\% to $13 \%$ of all patients with hypertension) for primary aldosteronism.

Since Conn described the first case of an aldosteroneproducing adenoma, several subtypes of primary aldosteronism have been described (Table 2). The differential diagnosis of these subtypes is crucial for the management and the prognosis.

\section{Clinical Presentation}

The diagnosis of primary aldosteronism is usually made in patients who are in the third to sixth decade of life. In cases associated with hypokalemia, patients may have muscle spasms, weakness, headaches, palpitations, polyuria, polydipsia, nocturia, or a combination of these. Periodic paralysis is a very rare presentation in Caucasians but not an infrequent presentation in patients of Asian descent. Sometimes, tetany can develop due to decreased ionized calcium from hypokalemic alkalosis. The nocturia and polyuria are a result of hypokalemia-induced renal concentrating defect, 
and the presentation is frequently mistaken for prostatic disease in men.

There are no specific physical findings. Edema is not a common finding because of the mineralocorticoid escape phenomenon. The degree of hypertension may be moderate to severe and may be resistant to usual pharmacologic treatments. Patient with aldosterone producing adenoma tend to have higher blood pressure compared to patient with bilateral idiopathic hyperplasia. Hypokalemia is frequently absent; in some patients, the hypokalemia can become evident only with the addition of potassium-wasting diuretics, and some patients can develop profound hypokalemia with only small doses of diuretics. Because of a reset osmostat, the serum sodium concentration tends to be high normal or above the upper limit of normal. This clinical clue is very useful when initially assessing the potential for primary aldosteronism.

Patients with primary aldosteronism may be at higher risk than other patients with essential or secondary hypertension for target-organ damage of the heart and kidney. That can be explained from a direct effect of aldosterone on these organs.

\section{Screening for Primary Aldosteronism}

The diagnostic approach to primary aldosteronism can be considered in three phases: screening, confirmation, followed by subtype evaluation tests.

Physicians should always consider screening for primary aldosteronism in the following situations:

(1) hypertension and hypokalemia,

(2) resistant hypertension,

(3) adrenal incidentaloma and hypertension,

(4) onset of hypertension at a young age ( $<20$ years),

(5) severe hypertension ( $>160 \mathrm{~mm}$ systolic or $>100$ diastolic),

(6) secondary hypertension,

(7) profound hypokalemia with usual doses of potassium wasting diuretics.

The initial screening tests include the measurement of plasma aldosterone concentration and plasma renin activity and calculation of the PAC/PRA (ARR) ratio. Like all biochemical case detection tests, the ARR is not without false positives and negatives. Table 2 documents the effect of medications and conditions on the ARR. The ARR should, therefore, be regarded as a detection test only and should be repeated if the initial results are inconclusive or difficult to interpret because of suboptimal sampling conditions.

If a patient is already on any of the medications listed in Table 3, he can be switched over to medications $[15,16]$ that do not affect or affect minimally the ARR. In Table 4 are listed the antihypertensive medications that have minimal effects on plasma aldosterone levels and can be used to control hypertension during case finding and confirmatory testing for primary aldosteronism $[15,16]$.

\begin{tabular}{|c|c|c|c|}
\hline & PAC & PRA & PAC/PRA \\
\hline \multicolumn{4}{|l|}{ Medications } \\
\hline$\beta$-Adrenergic blockers & $\downarrow$ & $\downarrow \downarrow$ & $\uparrow(\mathrm{FP})$ \\
\hline Central $\alpha-2$ agonists & $\downarrow$ & $\downarrow \downarrow$ & $\uparrow(\mathrm{FP})$ \\
\hline NSAIDs & $\downarrow$ & $\downarrow \downarrow$ & $\uparrow(\mathrm{FP})$ \\
\hline $\mathrm{K}^{+}$-wasting diuretics & $\rightarrow \uparrow$ & $\uparrow \uparrow$ & $\downarrow(\mathrm{FN})$ \\
\hline $\mathrm{K}^{+}$-sparing diuretics & $\uparrow$ & $\uparrow \uparrow$ & $\downarrow(\mathrm{FN})$ \\
\hline ACE inhibitors & $\downarrow$ & $\uparrow \uparrow$ & $\downarrow(\mathrm{FN})$ \\
\hline $\mathrm{ARBs}$ & $\downarrow$ & $\uparrow \uparrow$ & $\downarrow(\mathrm{FN})$ \\
\hline $\mathrm{Ca}^{2+}$ blockers (DHPs) & $\rightarrow \downarrow$ & $\uparrow$ & $\downarrow(\mathrm{FN})$ \\
\hline \multirow[t]{2}{*}{ Renin inhibitors } & $\downarrow$ & $\downarrow \uparrow^{1}$ & $\uparrow(\mathrm{FP})^{1}$ \\
\hline & & & $\downarrow(\mathrm{FN})^{1}$ \\
\hline \multicolumn{4}{|l|}{ Potassium status } \\
\hline Hypokalemia & $\downarrow$ & $\rightarrow \uparrow$ & $\downarrow(\mathrm{FN})$ \\
\hline Potassium loading & $\uparrow$ & $\rightarrow \downarrow$ & $\uparrow(\mathrm{FP})$ \\
\hline \multicolumn{4}{|l|}{ Dietary sodium } \\
\hline Sodium restricted & $\uparrow$ & $\uparrow \uparrow$ & $\downarrow(\mathrm{FN})$ \\
\hline Sodium loaded & $\downarrow$ & $\downarrow \downarrow$ & $\uparrow(\mathrm{FP})$ \\
\hline Advancing age & $\downarrow$ & $\downarrow \downarrow$ & $\uparrow(\mathrm{FP})$ \\
\hline \multicolumn{4}{|l|}{ Other conditions } \\
\hline Renal impairment & $\rightarrow$ & $\downarrow$ & $\uparrow(\mathrm{FP})$ \\
\hline PHA-2 & $\rightarrow$ & $\downarrow$ & $\uparrow(\mathrm{FP})$ \\
\hline Pregnancy & $\uparrow$ & $\uparrow \uparrow$ & $\downarrow(\mathrm{FN})$ \\
\hline Renovascular HTN & $\uparrow$ & $\uparrow \uparrow$ & $\downarrow(\mathrm{FN})$ \\
\hline Malignant HTN & $\uparrow$ & $\uparrow \uparrow$ & $\downarrow(\mathrm{FN})$ \\
\hline
\end{tabular}

The ARR is most sensitive when used in patients from whom samples are collected in the morning after patients have been out of bed for at least $2 \mathrm{~h}$, usually after they have been seated for 5-15 min and without posture stimulation. Ideally, patients should have unrestricted dietary salt intake before testing. In many cases, the ARR can be confidently interpreted with knowledge of the effect on the ARR of continued medications or suboptimal conditions of testing, avoiding delay and allowing the patient to proceed directly to confirmatory/exclusion testing $[15,16]$. Washout of all interfering antihypertensive medications is feasible in patients with mild hypertension but is potentially problematic in others and perhaps unnecessary in that medications with minimal effect on the ARR can be used in their place (Table 4).

A suggested approach $[15,16]$ for measurement of ARR as per the 2008 Endocrine Society guidelines follow.

\section{(A) Preparation for ARR Measurement: Agenda}

(1) Attempt to correct hypokalemia, after measuring plasma potassium in blood collected slowly with a syringe and needle (preferably not a Vacutainer to minimize the risk of spuriously raising potassium); avoid fist clenching during collection; wait at least $5 \mathrm{sec}$ after tourniquet release (if used to achieve insertion of needle); ensure separation of plasma from cells within 30 min of collection. 
TABLE 4

\begin{tabular}{|c|c|c|c|}
\hline Drug & Class & Usual dose & Comments \\
\hline Verapamil slow release & $\begin{array}{l}\text { Nondihydropyridine } \\
\text { calcium channel } \\
\text { antagonist }\end{array}$ & 90-120 mg twice daily & $\begin{array}{l}\text { Use singly or in combination } \\
\text { with the other agents listed in } \\
\text { this table. }\end{array}$ \\
\hline Hydralazine & Vasodilator & $\begin{array}{l}10-12.5 \mathrm{mg} \text { twice daily, } \\
\text { increasing as required }\end{array}$ & $\begin{array}{l}\text { Commence verapamil slow } \\
\text { release first to prevent reflex } \\
\text { tachycardia. Commencement at } \\
\text { low doses reduces risk of side } \\
\text { effects (including headaches, } \\
\text { flushing, and palpitations). }\end{array}$ \\
\hline Prazosin hydrochloride & $\alpha$-Adrenergic blocker & $\begin{array}{l}0.5-1 \mathrm{mg} \text { two to three } \\
\text { times daily, increasing as } \\
\text { required }\end{array}$ & $\begin{array}{l}\text { Monitor for postural } \\
\text { hypotension }\end{array}$ \\
\hline Doxazosin mesylate & $\alpha$-Adrenergic blocker & $\begin{array}{l}1-2 \mathrm{mg} \text { once daily, } \\
\text { increasing as required }\end{array}$ & $\begin{array}{l}\text { Monitor for postural } \\
\text { hypotension }\end{array}$ \\
\hline Terazosin hydrochloride & $\alpha$-Adrenergic blocker & $\begin{array}{l}1-2 \mathrm{mg} \text { once daily, } \\
\text { increasing as required }\end{array}$ & $\begin{array}{l}\text { Monitor for postural } \\
\text { hypotension }\end{array}$ \\
\hline
\end{tabular}

(2) Encourage patient to liberalize (rather than restrict) sodium intake.

(3) Withdraw agents that markedly affect the ARR (48) for at least 4 weeks:

(a) spironolactone, eplerenone, amiloride, and triamtereneb,

(b) potassium-wasting diureticsc,

(c) products derived from licorice root (e.g., confectionary licorice, chewing tobacco).

(4) If the results of ARR of the above agents are not diagnostic, and if hypertension can be controlled with relatively noninterfering medications (see Table 4 ), withdraw other medications that may affect the ARR (48) for at least 2 weeks like

(a) $\beta$-Adrenergic blockers, central $\alpha-2$ agonists (e.g., clonidine and $\alpha$-methyldopa), nonsteroidal anti-inflammatory drugs,

(b) angiotensin-converting enzyme inhibitors, angiotensin receptor blockers, renin inhibitors, and dihydropyridine calcium channel antagonists.

(5) If necessary to maintain hypertension control, commence other antihypertensive medications that have lesser effects on the ARR (e.g., verapamil slow release, hydralazine (with verapamil slow release, to avoid reflex tachycardia), prazosin, doxazosin, and terazosin; see Table 4).

(6) Establish OC and HRT status, because estrogencontaining medications may lower DRC and cause false-positive ARR when DRC (rather than PRA) is measured. Do not withdraw OC unless confident of alternative effective contraception.

\section{(B) Conditions for Collection of Blood}

(1) Collect blood mid-morning, after the patient has been up (sitting, standing, or walking) for at least $2 \mathrm{~h}$ and seated for 5-15 min.

(2) Collect blood carefully, avoiding stasis and hemolysis (see A.1 above).

(3) Maintain sample at room temperature (and not on ice, because this will promote conversion of inactive to active renin) during delivery to laboratory and before centrifugation and rapid freezing of plasma component pending assay.

(C) Factors to Take into Account when Interpreting Results (see Table 3)

(1) Age: in patients aged $>65 \mathrm{yr}$, renin can be lowered more than aldosterone by age alone, leading to a raised ARR.

(2) Time of day, recent diet, posture, and length of time in that posture.

(3) Medications.

(4) Method of blood collection, including any difficulty doing so.

(5) Level of potassium.

Level of creatinine (renal failure can lead to false-positive ARR).

Another important consideration when we are screening patients for primary aldosteronism is the reliability and the sensitivity of the assay we use for both aldosterone and plasma renin activity. Because the ARR is mathematically highly dependent on renin, renin assays should be sufficiently sensitive to measure levels as low as 0.2 $0.3 \mathrm{ng} / \mathrm{mL} \cdot \mathrm{h}$ (DRC $2 \mathrm{mU} /$ liter). Although most laboratories use radioimmunoassay (RIA) for plasma and urinary aldosterone, measured levels of standards have been shown to 
be unacceptably different in some instances. Tandem mass spectrometry is increasingly used and has proved to be much more consistent in performance.

\section{Interpretation of ARR}

Secondary hyperaldosteronism should be considered when both PRA and PAC are increased and the ARR is less than 10 (e.g., Renovascular disease). If both PAC and PRA are suppressed, an alternate source of mineralocorticoid receptor agonist should be considered (e.g., hypercortisolism). Primary aldosteronism should be suspected when PRA is suppressed $(<1 \mathrm{ng} / \mathrm{mL} / \mathrm{hr})$ and PAC is increased. It is important to understand that the lower limit of detection varies among different PRA assays and can have dramatic effect on the PAC/PRA ratio. For example, if the lower limit for detection of for PRA is $0.6 \mathrm{ng} / \mathrm{mL} / \mathrm{hr}$ and the PAC is $16 \mathrm{ng} / \mathrm{dL}$, then the ARR would be 27 . However, if the lower limit for detection of PRA is $0.1 \mathrm{ng} / \mathrm{mL} / \mathrm{hr}$, then the ARR would be 160 . Thus, the ARR cutoff for a high ARR is laboratory dependent and more specifically PRA assay dependent.

In a retrospective study [17], the combination of an ARR $>30$ and PAC $>20 \mathrm{ng} / \mathrm{dL}$ had a sensitivity of $90 \%$ and a specificity of $91 \%$ for aldosterone producing adenomas. At Mayo clinic, an ARR of more than 20 and a PAC $>15 \mathrm{ng} / \mathrm{dL}$ are found in more than $90 \%$ of patients with surgically confirmed aldosterone producing adenomas.

Most groups, however, use cutoffs of 20-40 when testing is performed in the morning on a seated ambulatory patient. Some investigators [18] require elevated aldosterone levels in addition to elevated ARR for a positive screening test for PA (usually aldosterone $>15 \mathrm{ng} / \mathrm{dL}$.) An alternative approach is to avoid a formal cutoff level for plasma aldosterone but to recognize that the likelihood of a false-positive ARR becomes greater when renin levels are very low.

Against a formal cutoff level for aldosterone are the findings of several studies. In one study, seated plasma aldosterone levels were less than $15 \mathrm{ng} / \mathrm{d}$ in $36 \%$ of 74 patients diagnosed with PA after screening positive by ARR defined as more than 30 and showing failure of aldosterone to suppress during fludrocortisone suppression testing (FST) and in four of 21 patients found by AVS to have unilateral, surgically correctable PA. Another study reported plasma aldosterone levels of 9-16 ng/dL in 16 of 37 patients diagnosed with PA by FST. Although it would clearly be desirable to provide firm recommendations for ARR and plasma aldosterone cutoffs, the variability of assays between laboratories and the divided literature to date makes it more prudent to point out relative advantages and disadvantages, leaving clinicians the flexibility to judge for themselves.

\section{Confirmatory Tests}

An increased ARR is not diagnostic by itself and confirmatory tests are required to demonstrate inappropriate aldosterone secretion. Treatment with spironolactone or eplerenone should not be started before the confirmation of primary aldosteronism. In patients already receiving treatment with spironolactone or eplerenone, the treatment should be held for at least 4-6 weeks before further diagnostic testing. Aldosterone suppression testing can be performed with orally administered sodium chloride and measurement of urinary aldosterone or with intravenous sodium chloride loading and measurement of PAC.

\section{Oral Sodium Loading Test}

After hypertension and hypokalemia are controlled, patients should increase their sodium intake to $>200 \mathrm{mmol} / \mathrm{d}$ $(\sim 6 \mathrm{~g} / \mathrm{d})$ for $3 \mathrm{~d}$, verified by 24 -h urine sodium content. Patients should receive adequate slow-release potassium chloride supplementation to maintain plasma potassium in the normal range. Urinary aldosterone is measured in the 24$\mathrm{h}$ urine collection from the morning of $\mathrm{d} 3$ to the morning of d 4. Primary aldosteronism is unlikely if urinary aldosterone is lower than $10 \mu \mathrm{g} / 24 \mathrm{~h}(27.7 \mathrm{nmol} / \mathrm{d})$ in the absence of renal disease, where primary aldosteronism may coexist with lower measured urinary aldosterone levels. Elevated urinary aldosterone excretion $(>12 \mu \mathrm{g} / 24 \mathrm{~h}(>33.3 \mathrm{nmol} / \mathrm{d})$ at the Mayo Clinic, $>14 \mu \mathrm{g} / 24 \mathrm{~h}(38.8 \mathrm{nmol} / \mathrm{d})$ at the Cleveland Clinic) makes primary aldosteronism highly likely. This test should not be performed in patients with severe uncontrolled hypertension, renal insufficiency, cardiac insufficiency, cardiac arrhythmia, or severe hypokalemia. The 24-h urine collection may be inconvenient. Laboratory-specific poor performance of the RIA for urinary aldosterone (aldosterone 18-oxo-glucuronide or acid-labile metabolite) may blunt diagnostic accuracy, a problem obviated by the currently available HPLC-tandem mass spectrometry methodology. Aldosterone 18-oxo-glucuronide is a renal metabolite, and its excretion may not rise in patients with renal disease.

\section{Intravenous Saline Infusion Test}

The test is done after an overnight fast. Patients stay in the recumbent position for at least $1 \mathrm{~h}$ before and during the infusion of 2 liters of $0.9 \%$ saline iv over $4 \mathrm{~h}$, starting at 0800 0930 h. Blood samples for renin, aldosterone, cortisol, and plasma potassium are drawn at time zero and after $4 \mathrm{~h}$, with blood pressure and heart rate monitored throughout the test. Postinfusion plasma aldosterone levels $<5 \mathrm{ng} / \mathrm{dL}$ make the diagnosis of primary aldosterone unlikely, and levels $>10 \mathrm{ng} / \mathrm{dL}$ are a very probable sign of primary aldosterone. Values between 5 and $10 \mathrm{ng} / \mathrm{dL}$ are indeterminate and can be seen in patients with bilateral idiopathic hyperplasia. This test should not be performed in patients with severe uncontrolled hypertension, renal insufficiency, cardiac insufficiency, cardiac arrhythmia, or severe hypokalemia.

\section{Subtype Studies}

All patients with primary aldosteronism (PA) should undergo an adrenal CT scan as the initial study in subtype testing and to exclude large masses that may represent adrenocortical carcinoma. The findings on adrenal 
CT-normal-appearing adrenals, unilateral macroadenoma $(>1 \mathrm{~cm})$, minimal unilateral adrenal limb thickening, unilateral microadenomas $(\leq 1 \mathrm{~cm})$, or bilateral macro- or microadenomas (or a combination of the two)-are used in conjunction with adrenal venous sampling (AVS) and, if needed, ancillary tests to guide treatment decisions in patients with PA. Aldosterone-producing adenomas (APA) may be visualized as small hypodense nodules (usually $<2 \mathrm{~cm}$ in diameter) on CT. Adrenal glands with bilateral idiopathic hyperplasia (IHA) may be normal on CT or show nodular changes. Aldosterone-producing adrenal carcinomas are almost always more than $4 \mathrm{~cm}$ in diameter, but occasionally smaller, and like most adrenocortical carcinomas have a suspicious imaging phenotype on CT.

Adrenal CT has several limitations. Small APAs may be interpreted incorrectly by the radiologist as IHA on the basis of CT findings of bilateral nodularity or normalappearing adrenals. Moreover, apparent adrenal microadenomas may actually represent areas of hyperplasia, and unilateral adrenalectomy would be inappropriate. In addition, nonfunctioning unilateral adrenal macroadenomas are not uncommon, especially in older patients $(>40 \mathrm{yr})$ and are indistinguishable from APAs on CT. Unilateral UAH may be visible on $\mathrm{CT}$, or the $\mathrm{UAH}$ adrenal may appear normal on CT. Magnetic resonance imaging has no advantage over CT in subtype evaluation of PA, being more expensive and having less resolution than $\mathrm{CT}$.

\section{Adrenal Venous Sampling}

Lateralization of the source of the excessive aldosterone secretion is critical to guide the management of PA. Distinguishing between unilateral and bilateral disease is important because unilateral adrenalectomy in patients with APA or UAH results in normalization of hypokalemia in all; hypertension is improved in all and cured in 30\%$60 \%[19,20]$. In bilateral IHA and GRA, unilateral or bilateral adrenalectomy seldom corrects the hypertension, and medical therapy is the treatment of choice. Unilateral disease may be treated medically if the patient declines or is not a candidate for surgery.

Adrenal vein sampling was initially proposed as a way to localize catecholamine secreting neoplasms (Young 2009). In 1967, Melby et al. reported the use of adrenal vein sampling in primary aldosteronism, and it has since become the gold standard test to differentiate unilateral from bilateral disease (Young 2009). In his initial paper, he reported the comparison of aldosterone from the right and left adrenal veins in seven patients with primary aldosteronism and also in three normal patients. The authors found elevated aldosterone secretion and a higher aldosterone to cortisol ratio from the involved adrenal gland compared to the uninvolved adrenal gland. All primary aldosteronism patients in the study were operated for unilateral adrenalectomy and were found to have adenomas that correlated with the lateralization found on adrenal vein sampling (Melby 1967).
From this first report, adrenal vein sampling in primary aldosteronism has continued to evolve. Some controversies remain including whether the addition of cosyntropin to the test either as a bolus or constant infusion improves the diagnostic accuracy and what diagnostic criteria to use (Auchus 2010). In performing this test, there are some things that need to be considered. First, the patient needs to be prepared properly. They should be tested in a fasting state and be supine for at least one hour before testing (Young 2009). Additionally, certain antihypertensives should be avoided to prevent false results. Mineralocorticoid receptor antagonists in particular should be discontinued at least 6 weeks before testing. Preferred medications include extended release verapamil, $\alpha$-adrenergic blockers, and hydralazine. Patients should also be potassium repleted as hypokalemia can lower the aldosterone levels (Rossi 2007).

In addition to patient preparation, the test should be performed by an experienced and skilled practitioner, especially given some of the technical difficulties that can arise in cannulating the right adrenal vein. Additionally, a well-defined protocol should be in place (Rossi 2007, Young 2009). Samples are obtained from the right adrenal, left adrenal, and from a peripheral source. This should be performed simultaneously or in rapid sequence to limit fluctuations in secretion that occur with time (Young 2009).

The NIH protocol for adrenal vein sampling in primary aldosteronism is as follows.

(1) Catheterization of bilateral femoral veins is performed with a 0.45 polyurethane catheter with 6 French sheath in the right, and Muller catheter in the left.

(2) Cannulation of both adrenal veins is obtained via the right femoral vein.

(3) Simultaneous baseline sampling for aldosterone and cortisol is performed from both adrenal veins, and a peripheral sample is drawn from the left femoral catheter at time -5 minutes and at time 0 minutes.

(4) After obtaining baseline samples, a $0.25 \mathrm{mg}$ push of ACTH is given followed by steady infusion of ACTH of $1 \mathrm{mcg} / \mathrm{ml}$ at $150-200 \mathrm{ml} / \mathrm{hr}$.

(5) At time +10 minutes and +15 minutes, simultaneous samples are again obtained from right adrenal vein, left adrenal vein, and left femoral vein.

(6) Upon completion of sampling, ACTH infusion is stopped and switched to normal saline at $\mathrm{KVO}$, and catheters are removed.

It is important to note that all samples are stored on ice during sampling and are then transported to the laboratory on ice for processing.

Interpretation of the results of the sampling is dependent not only on the aldosterone concentration and on the cortisol concentration but also on whether ACTH stimulation was used. In the setting of ACTH stimulation during adrenal vein sampling, a few ratios need to be calculated. The first is the selectivity index (SI) which is used to determine if there was adequate cannulation of the adrenal veins. This is calculated 
TABLe 5

\begin{tabular}{lcccc}
\hline Location & Aldosterone & Cortisol & Selectivity index & A : C ratio \\
\hline Right adrenal & A & D & D/F & A/D \\
Left adrenal & B & E & E/F & B/E \\
Peripheral & C & F & & \\
\hline
\end{tabular}

by taking the ratio of cortisol from the adrenal compared to the cortisol from the peripheral sample. This should be done for both right and left sided samples. With ACTH stimulation, a ratio of $5: 1$ indicates successful cannulation of the adrenal vein. Without ACTH stimulation, a ratio of $3: 1$ suggests successful cannulation though different cut-offs have been suggested (Young 2009).

After confirming successful cannulation, the next step is to determine unilaterality versus bilaterality. First, the aldosterone concentration needs to be corrected for possible dilution by dividing by the cortisol to get the $\mathrm{A}$ : $\mathrm{C}$ ratio. Next, the cortisol corrected values are compared to calculate the lateralization index (LI). The greater of A: C ratio is divided by the smaller $\mathrm{A}$ : $\mathrm{C}$ ratio. A ratio of $>4: 1$ suggests lateralization, <3:1 suggests bilateral disease, and anything in between is inconclusive (Young 2009, Auchus 2010). An example of the calculation follows Table 5 .

In this example, selectivity index (SI) should be $>5$ to indicate successful cannulation of the adrenal veins. Lateralization index (LI) would be calculated by taking the higher $\mathrm{A}: \mathrm{C}$ ratio divided by the lower $\mathrm{A}: \mathrm{C}$ ratio. If $\mathrm{LI}$ is $>4$, then unilateral disease lateralizing to the numerator side is suggested. If LI is $<3$, then bilateral disease is suggested. If LI is between 3 and 4 , then the results are indeterminate and a repeat study may need to be considered. Alternatively, repeating the assay on samples stored from the test or measuring 18-hydroxycorticosterone could be considered (Auchus 2010). The true sensitivity and specificity of the test is difficult to determine, as calculating this would require performing an adrenalectomy on all patients to verify the diagnosis.

\section{Treatment}

Unilateral laparoscopic adrenalectomy should be offered to patients with documented unilateral PA (i.e., APA or UAH). If a patient is unable or unwilling to undergo surgery, medical treatment with a mineralocorticoid receptor antagonist is recommended. Hypertension is cured (defined as blood pressure $<140 / 90 \mathrm{~mm} \mathrm{Hg}$ without the aid of antihypertensive drugs) in about $50 \%$ (range, $35 \%-60 \%$ ) of patients with APA after unilateral adrenalectomy, with a cure rate as high as $56 \%-77 \%$ when the cure threshold was blood pressure less than $160 / 95 \mathrm{~mm} \mathrm{Hg}$.

There are two mineralocorticoid receptor antagonists that can be used for the management of patients with primary hyperaldosteronism: spironolactone and eplerenone. Usually, a low dose of either antagonist can significantly and effectively improve hypokalemia, but for the management of hypertension, high doses are required. In patients intolerant to mineralocorticoid receptor antagonists, dihydropyridine calcium channel blockers such as nifedipine can be used alternatively, since studies have been shown that it directly inhibits the synthesis of aldosterone and blocks the mineralocorticoid receptors.

Factors associated with resolution of hypertension in the postoperative period include having one or no firstdegree relative with hypertension and preoperative use of two or fewer antihypertensive drugs. Other factors have been reported to predict cure but have been evaluated by only univariate analysis or when the cutoff for blood pressure resolution was less than $160 / 95 \mathrm{~mm} \mathrm{Hg}$, duration of hypertension less than $5 \mathrm{yr}$, higher PAC to PRA ratio preoperatively, higher urinary aldosterone secretion, or positive preoperative response to spironolactone. The most common reasons for persistently increased blood pressure after adrenalectomy are coexistent hypertension of unknown cause and older age and/or longer duration of hypertension.

\section{Pre- and Postoperative Management}

In the patient scheduled for surgery, both hypertension and hypokalemia should be well controlled preoperatively. Obtaining such control may require a delay in surgery and the addition of a mineralocorticoid receptor antagonist.

Plasma aldosterone and renin activity levels should be measured shortly after surgery as an early indication of biochemical response, and on postoperative day 1 , potassium supplementation should be withdrawn, spironolactone discontinued, and antihypertensive therapy reduced, if appropriate.

Postoperative iv fluids should be normal saline without potassium chloride unless serum potassium levels remain very low (i.e., $<3.0 \mathrm{mmol} / \mathrm{liter}$ ), and during the first few weeks after surgery, a generous sodium diet should be recommended to avoid the hyperkalemia that can develop from hypoaldosteronism due to chronic contralateral adrenal gland suppression. In rare instances, temporary fludrocortisone therapy may be required.

Blood pressure typically normalizes or shows maximal improvement in 1-6 months after unilateral adrenalectomy for unilateral APA but can continue to fall for up to $1 \mathrm{yr}$ in some patients.

\section{Familial Forms of Primary Aldosteronism}

Glucocorticoid-remediable aldosteronism (GRA), also known as familial hyperaldosteronism type I (FH I), is a monogenic form of inherited hypertension, first described in 1966 by Sutherland et al. (1966). This disease is characterized by high plasma aldosterone levels, suppressed plasma renin activity, and abnormally high production of two rare steroids: 18-hydroxycortisol (18OHF) and 18-oxocortisol (180xoF). The synthesis of these steroids requires the simultaneous presence of a 17-hydroxylase activity and the two C18 (18-hydroxylase and 18-oxidase) activities typical of the CYP11B2 (aldosterone synthase) enzyme.

In GRA, the secretion of aldosterone is primarily regulated by adrenocorticotropic hormone (ACTH) rather than 
angiotensin II; in fact, the symptoms are exacerbated by ACTH administration and normalized by glucocorticoid administration. Despite the state of hyperaldosteronism, hypokalemia is not a common feature. In affected families, there is an increased frequency of early death from stroke and an increased risk for exacerbation of hypertension during pregnancy. However, the majority of affected family members has mild-to-moderate hypertension and normal biochemistry and is clinically indistinguishable from patients with essential hypertension. It is, therefore, possible that this condition is under diagnosed. This monogenic form of hypertension is noteworthy, because it is frequently unresponsive to standard antihypertensive medication but successfully managed by treatment with amiloride, spironolactone, or dexamethasone alone.

The GRA is inherited in an autosomal dominant fashion and is caused by the presence of a chimeric gene originating from an unequal crossover between the CYP11B1 (11_-hydroxylase) and CYP11B2 genes. The hybrid gene has the CYP11B1 sequence at the $5^{\prime}$ end, including the promoter, and the CYP11B2 sequence at the $3^{\prime}$ end. The $C Y P 11 B 1$ promoter ensures the expression of the hybrid gene throughout the adrenal cortex, whereas the CYP11B2 sequence leads to the inappropriate synthesis of aldosterone, $18 \mathrm{OHF}$, and $18 \mathrm{oxoF}$. The exact position of the crossover site, occurring between intron 2 and exon 4 , does not seem to affect the phenotype. Aldosterone suppression by dexamethasone, and high $18 \mathrm{OHF}$ and $18 \mathrm{oxoF}$ levels are used to differentiate glucocorticoid-remediable aldosteronism from the other forms of primary aldosteronism. Definitive diagnosis can only be reached by identification of the CYP11B1/CYP11B2 chimeric gene in genomic DNA using either Southern blotting or the long PCR technique.

FH-II is an inherited, nonglucocorticoid remediable form of hyperaldosteronism that was relatively recently recognized as a distinct entity. The onset of FH-II occurs usually in adulthood. Apart from its familial occurrence, FH-II is clinically, biochemically, and morphologically indistinguishable from apparently nonfamilial primary aldosteronism and also overlaps with essential hypertension. Pathologic aldosterone secretion may result from unilateral adenoma or bilateral hyperplasia with different subtypes sometimes occurring within different members of the same family. Diagnosis of FH-II can be suspected by documenting primary aldosteronism in two-to-three members (ideally two generations) of a family and excluding the CYP11B1/2 gene. Most, but not all patients could be linked to chromosome $7 \mathrm{p} 22$, consistent with the hypothesis that FH-II and maybe primary aldosteronism in general are likely to be genetically heterogeneous [21, 21$]$. Since FH-II lacks steroid sensitivity, therapeutic options resemble those of the sporadic forms of primary aldosteronism. Like in FH$\mathrm{I}$ it is advisable to avoid genomic and nongenomic effects by removal of the source of aldosterone secretion. Of course, surgery is only indicated if lateralization can be demonstrated by adrenal vein sampling. The second-best approach is blockage of aldosterone effects with receptor antagonists.

\section{Summary}

Primary hyperaldosteronism is more frequent in hypertensive subjects than was previously believed. It is now recognized that the APA is just one of the seven subtypes of primary aldosteronism. APA and bilateral idiopathic hyperaldosteronism (IHA) are the most common subtypes of primary aldosteronism. Using the plasma aldosterone to plasma renin activity ratio as a case-finding test, followed by aldosterone suppression confirmatory testing, has resulted in much higher prevalence estimates of $5 \%-13 \%$ of all patients with hypertension. In patients with suspected primary aldosteronism, screening can be accomplished by measuring a morning (preferably between 0800 and $1000 \mathrm{~h}$ ) ambulatory paired random plasma aldosterone concentration (PAC) and plasma renin activity (PRA). An increased PAC: PRA ratio is not diagnostic by itself, and primary aldosteronism must be confirmed by demonstrating inappropriate aldosterone secretion. Aldosterone suppression testing can be performed with orally administered sodium chloride and measurement of urinary aldosterone or with intravenous sodium chloride loading and measurement of PAC. Adrenal venous sampling performed by an experienced interventional radiologist is the gold standard for the differentiation between APA and non-APA-causes of primary aldosteronism. Distinguishing between unilateral and bilateral adrenal hypersecretion is critical in assessing treatment options.

\section{Acknowledgment}

This work was supported by the Intramural Program of the Eunice Kennedy Shriver National Institute of Child Health and Human Development.

\section{References}

[1] J. Muller and M. Lauber, "Regulation of aldosterone biosynthesis: a continual challenge," American Journal of Hypertension, vol. 4, pp. 280-282, 1991.

[2] J. Muller, "Final steps of aldosterone biosynthesis: molecular solution of a physiological problem," Journal of Steroid Biochemistry and Molecular Biology, vol. 45, no. 1-3, pp. 153159, 1993.

[3] K. M. Curnow, M. T. Tusie-Luna, L. Pascoe et al., "The product of the CYP11B2 gene is required for aldosterone biosynthesis in the human adrenal cortex," Molecular Endocrinology, vol. 5, no. 10, pp. 1513-1522, 1991.

[4] D. W. Russell and P. C. White, "Four is not more than two," American Journal of Human Genetics, vol. 57, no. 5, pp. 10021005, 1995.

[5] S. E. Taymans, S. Pack, E. Pak, D. J. Torpy, Z. Zhuang, and C. A. Stratakis, "Human CYP11B2 (Aldosterone synthase) maps to chromosome 8q24.3," Journal of Clinical Endocrinology and Metabolism, vol. 83, no. 3, pp. 1033-1036, 1998.

[6] T. J. McKenna, D. P. Island, W. E. Nicholson, and G. W. Liddle, "The effect of potassium on early and late steps in aldosterone biosynthesis in cells of the zona glomerulosa," Endocrinology, vol. 103, no. 4, pp. 1411-1416, 1978.

[7] C. D. Clyne, Y. Zhang, L. Slutsker, J. M. Mathis, P. C. White, and W. E. Rainey, "Angiotensin II and potassium 
regulate human CYP11B2 transcription through common ciselements," Molecular Endocrinology, vol. 11, no. 5, pp. 638649, 1997.

[8] D. J. Torpy, C. A. Stratakis, and G. P. Chrousos, "Hyper- and hypoaldosteronism," Vitamins and hormones, vol. 57, pp. 177216, 1999.

[9] J. J. Brown, D. L. Davies, A. F. Lever, and J. I. Robertson, "Variations in plasma renin concentration in several physiological and pathological states," Canadian Medical Association journal, vol. 90, pp. 201-206, 1964.

[10] J. R. Blair-West, J. P. Coghlan, and D. A. Denton, "A doseresponse comparison of the actions of angiotensin II and angiotensin III in sheep," Journal of Endocrinology, vol. 87, no. 3, pp. 409-417, 1980.

[11] T. Kono, F. Oseko, and F. Ikeda, "Biological activity of des asp, ileu $^{8}$ angiotensin II (ileu ${ }^{8}$ angiotensin III) in man," Journal of Clinical Endocrinology and Metabolism, vol. 43, no. 4, pp. 940943, 1976.

[12] R. M. Carey, "Acute dopaminergic inhibition of aldosterone secretion is independent of angiotensin II and adrenocorticotropin," Journal of Clinical Endocrinology and Metabolism, vol. 54, no. 2, pp. 463-469, 1982.

[13] S. Kapas and J. P. Hinson, "Adrenomedullin in the adrenal," Microscopy Research and Technique, vol. 57, no. 2, pp. 91-97, 2002.

[14] J. P. Hinson, J. R. Puddefoot, and S. Kapas, "Actions of vasoactive intestinal peptide on the rat adrenal zona glomerulosa," Journal of Endocrinology, vol. 161, no. 1, pp. 51-57, 1999.

[15] J. W. Funder, R. M. Carey, C. Fardella et al., "Case detection, diagnosis, and treatment ofpatients with primary aldosteronism: an endocrine society clinical practice guideline," Journal of Clinical Endocrinology and Metabolism, vol. 93, no. 9, pp. 3266-3281, 2008.

[16] M. H. Weinberger and N. S. Fineberg, “The Endocrine Society The diagnosis of primary aldosteronism and separation of two major subtypes," Archives of Internal Medicine, vol. 153, no. 18, pp. 2125-2129, 1993.

[17] W. F. Young, "Primary aldosteronism: renaissance of a syndrome," Clinical Endocrinology, vol. 66, no. 5, pp. 607-618, 2007.

[18] O. Celen, M. J. O’Brien, J. C. Melby, and R. M. Beazley, "Factors influencing outcome of surgery for primary aldosteronism," Archives of Surgery, vol. 131, no. 6, pp. 646-650, 1996.

[19] A. Meyer, G. Brabant, and M. Behrend, "Long-term followup after adrenalectomy for primary aldosteronism," World Journal of Surgery, vol. 29, no. 2, pp. 155-159, 2005.

[20] A. So, D. L. Duffy, R. D. Gordon et al., "Familial hyperaldosteronism type II is linked to the chromosome 7p22 region but also shows predicted heterogeneity," Journal of Hypertension, vol. 23, no. 8, pp. 1477-1484, 2005.

[21] A. R. Lafferty, D. J. Torpy, M. Stowasser et al., "A novel genetic locus for low renin hypertension: familial hyperaldosteronism type II maps to chromosome 7 (7p22)," Journal of Medical Genetics, vol. 37, no. 11, pp. 831-835, 2000. 


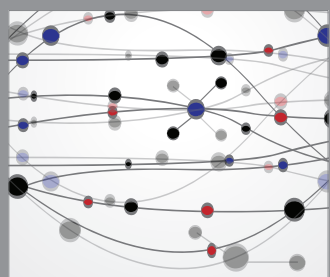

The Scientific World Journal
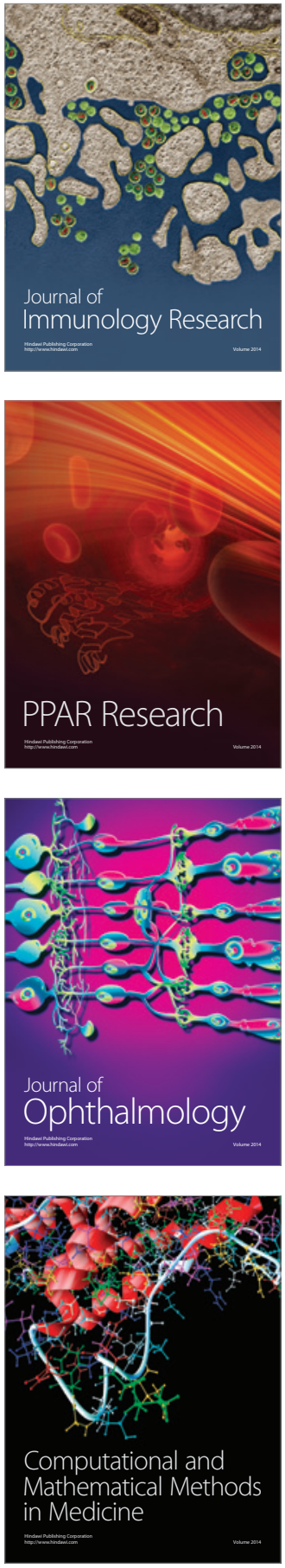

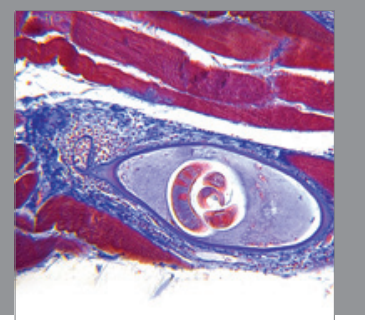

Gastroenterology

Research and Practice
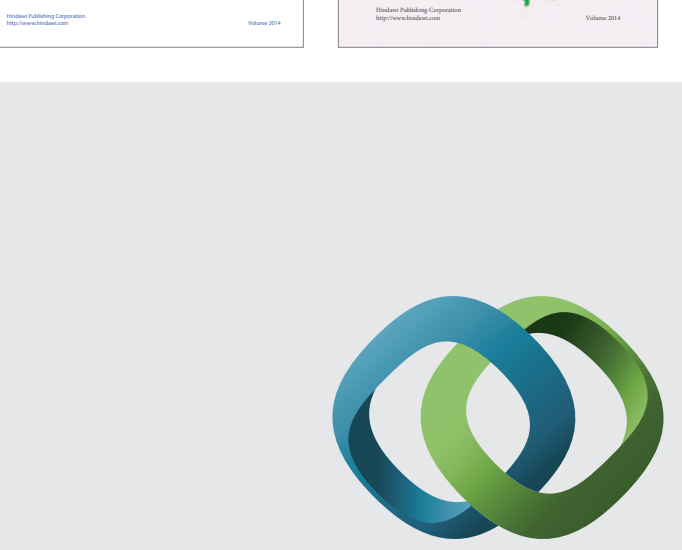

\section{Hindawi}

Submit your manuscripts at

http://www.hindawi.com
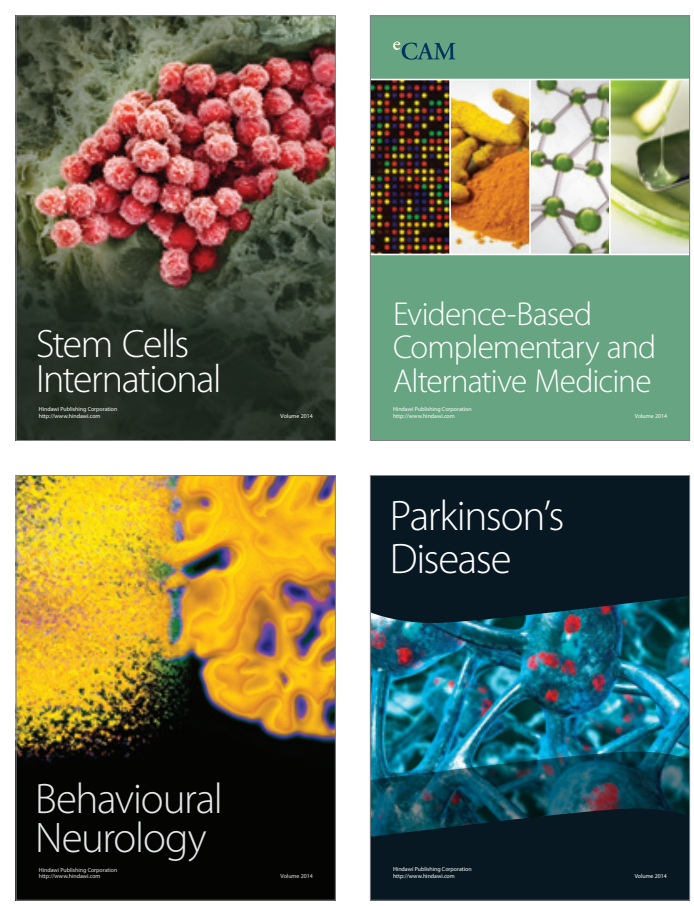

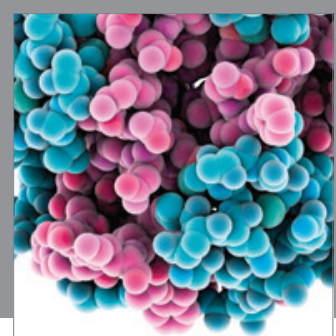

Journal of
Diabetes Research

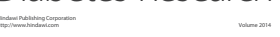

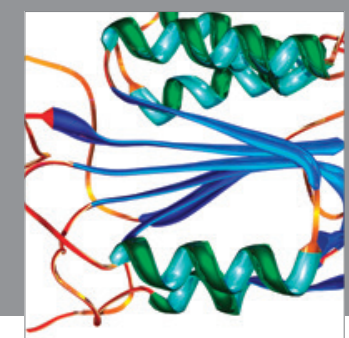

Disease Markers
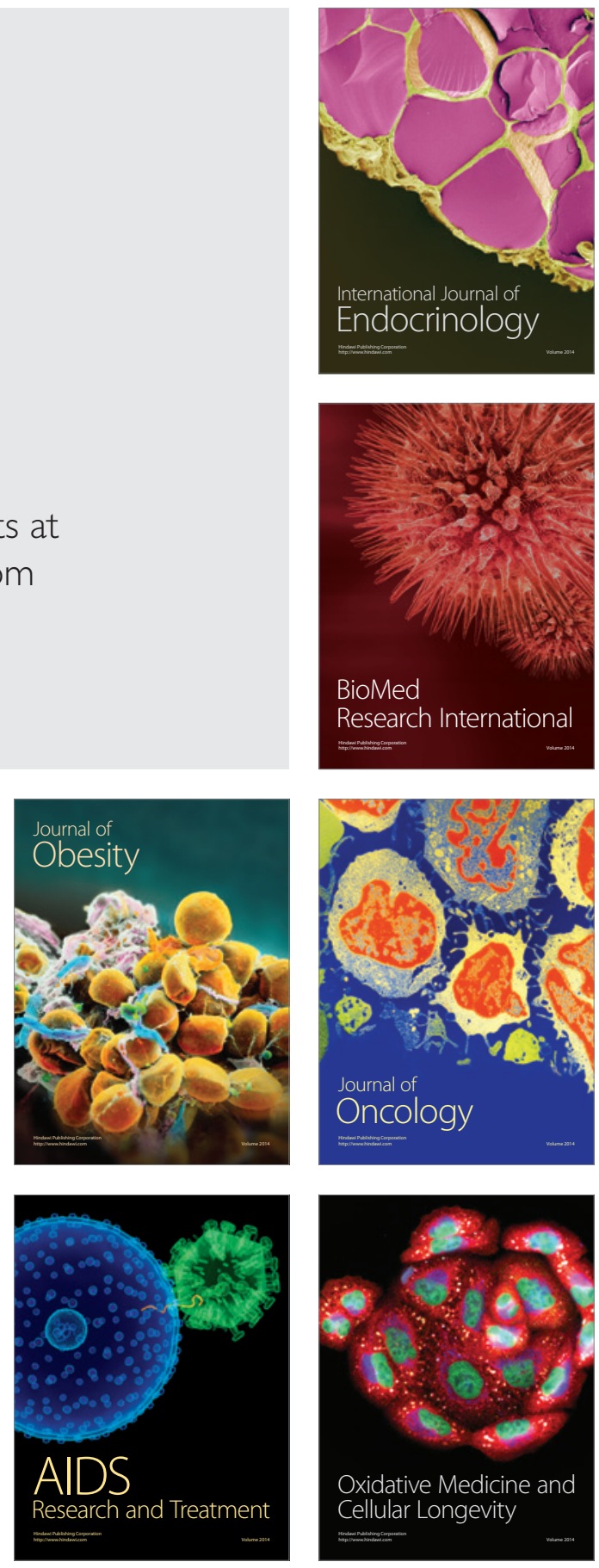